\title{
Approximate analytical solution for one-dimensional Richards' equation
}

\author{
Yuwei Zhang ${ }^{1, \text { a }}$, Xi Chen ${ }^{2}$ and Wei Luo ${ }^{2, b}$ \\ ${ }^{1}$ China Merchants Chongqing Communications Technology Research \& Design Institute CO.,LTD, \\ Chongqing 400067, China \\ ${ }^{2}$ College of Hydraulic \& Environmental Engineering, Three Gorges University, Yichang 443002 , \\ China \\ azhangyuwei1015@126.com, bwluo@Lzu.edu.cn
}

Keywords: approximate analytical solution, Richards' equation, Brooks-Corey model, Boltzmann transformation.

Abstract. It is derived an approximate analytical solution of one-dimensional Richards' equation (RE) with Brooks-Corey model in this paper. The approach is based on the introduction of Boltzmann transformation and construction of an intermediate variable for approximation. In the end, one illustration is given to confirm the accuracy of present solution.

\section{Introduction}

Richards' equation (RE) is a non-linear partial differential equation (PDE) of saturation and can be expressed as [1]

$\frac{\partial S}{\partial t}=\frac{\partial}{\partial x}\left(D(S) \frac{\partial S}{\partial x}\right)$

where $S$ is the saturation of unsaturated porous media [-], $x$ and $t$ are the space and the time coordinates, $D(S)$ are the diffusivity function $\left[\mathrm{L}^{2} / \mathrm{T}\right]$ of saturation. It is considered the infiltration of water in a semi-infinite domain $x \in[0,+\infty)$ and the boundary conditions are [2]

$\left.S\right|_{x=0}=S_{L},\left.S\right|_{x \rightarrow+\infty}=S_{0}$.

The initial condition is

$\left.S\right|_{t=0}=S_{0}$,

where $S_{0} \in[0,1]$ is an arbitrary constant.

In these years, much research such as Adomian decomposition method (ADM) [2], traveling wave method (TWM) [3], differential transform method (DTM) [4], homotopy perturbation method (HPM) [4], extend Heaslet \& Alksne technique and so on [5], has been done to derive the exact solution, which has a wide theoretical and practical applicability. These techniques are very powerful in solving complex non-linear problems. Influenced and motivated by research before, it is solved one-dimensional RE with arbitrary diffusivity and initial constant $S_{0}$ by Boltzmann transformation, which changes RE into a no-linear ordinary differential equation (ODE). Then an intermediate variable is introduced and term wise derivative is applied in obtaining an approximate solution of the ODE. At last, an example of Brooks-Corey model is used to test the accuracy of present solution.

\section{Model definition}

There are lots of models [5, 7] have been suggested for determining the diffusivity $D(S)$ in RE because the varied applications. The Brooks-Corey model is the more commonly used model among them [4]. In this section, we define the problem to be solved by introducing the Brooks-Corey model.

The diffusivity function of Brooks-Corey model is expressed by a power law as [5] 
$D(S)=D_{0} S^{n}$

where $D_{0}$ and $n$ are the empirically-fitted constants.

\section{Solving the RE}

Introducing a new saturation $S_{1}\left(=S-S_{0}\right)$, the initial condition Eq. 3 is simplified as

$\left.S_{1}\right|_{t=0}=0$,

and RE in Eq. 1 can be written as

$\frac{\partial S}{\partial t}=\frac{\partial}{\partial x}\left(D\left(S_{1}+S_{0}\right) \frac{\partial S_{1}}{\partial x}\right)$

The boundary conditions Eq. 2 are changed into

$$
\left.S_{1}\right|_{x=0}=S_{L}-S_{0},\left.S_{1}\right|_{x \rightarrow+\infty}=0 \text {. }
$$

According to Parlange ${ }^{[1]}$, the Boltzmann variable $\phi=x / \sqrt{t}$ is introduced, and Eq. 6 is transformed as $^{[1]}$

$-\frac{1}{2} \phi \frac{\mathrm{d} S_{1}}{\mathrm{~d} \phi}=\frac{\mathrm{d}}{\mathrm{d} \phi}\left(D\left(S_{1}+S_{0}\right) \frac{\mathrm{d} S_{1}}{\mathrm{~d} \phi}\right)$

Then the initial condition Eq. 5 and boundary conditions Eq. 7 are [5]

$\left.\phi\right|_{S_{1}=0} \rightarrow+\infty,\left.\phi\right|_{S_{1}=S_{L}-S_{0}}=0$.

At this time, RE 1 with the boundary conditions Eq. 2 and the initial condition Eq. 3 are changed into ODE Eq. 8 with the boundary conditions Eq. 9.

Integrating on Eq. 8, we obtain [5]

$\frac{\mathrm{d} \phi}{\mathrm{d} S_{1}} \int_{0}^{S_{1}} \phi \mathrm{d} S_{1}=-2 D\left(S_{1}+S_{0}\right)$

the boundary conditions remain to be Eq. 9. Here, we construct an intermediate variable [8]

$\xi=\int_{S_{1}}^{S_{L}-S_{0}} \frac{D\left(S_{1}+S_{0}\right)}{S_{1}} \mathrm{~d} S_{1}$,

and $n_{1}(=1,2,3, \ldots)$ order approximate solution of $\phi$ in Eq. 10 yields [8]

$\phi \approx \begin{cases}\sum_{i=1}^{n_{1}} U_{i}\left(\int_{S_{1}}^{S_{L}-S_{0}} \frac{D\left(S_{1}+S_{0}\right)}{S_{1}} \mathrm{~d} S_{1}\right)^{i}, & S_{1} \neq 0, \\ +\infty, & S_{1}=0\end{cases}$

which is satisfies the boundary conditions Eq. 9. In Eq. 12, $n_{1}$ and $U_{i}$ are parameters. Applying the term wise derivative approach, Eq. 10 can be written as

$\left.\frac{\mathrm{d}^{(k)}}{\mathrm{d} S_{1}^{(k)}}\left(\frac{\mathrm{d} \phi}{\mathrm{d} S_{1}} \int_{0}^{S_{1}} \phi \mathrm{d} S_{1}\right)\right|_{S_{1}=S_{L}-S_{0}}=-\left.2 \frac{\mathrm{d}^{(k)} D\left(S_{1}+S_{0}\right)}{\mathrm{d} S_{1}^{(k)}}\right|_{S_{1}=S_{L}-S_{0}}$, 
where $k=0,1,2,3, \ldots, n_{1}-1 . U_{i}$ can be calculated by substituting Eq. 12 into Eq. 13 and solving a series of non-linear algebraic equations.

\section{Numerical simulation}

In this section, an example of Brooks-Corey model is given to confirm the accuracy of present method. It is taken parameters as $D_{0}=247.1$ and $n=4$ [5] in Eq. 4, and the parameters $S_{0}$ and $S_{L}$ in Eqs. 2-3 are taken as $S_{0}=0$ and $S_{L}=1$ respectively [5]. Applying the approach in Eqs. 12-13, 1-5 order approximate solutions are shown as

1 order approximate solution

$\frac{x}{\sqrt{t}} \approx 12.4273\left(1-S^{4}\right)$.

2 order approximate solution

$\frac{x}{\sqrt{t}} \approx 13.1811\left(1-S^{4}\right)-1.6476\left(1-S^{4}\right)^{2}$.

3 order approximate solution

$\frac{x}{\sqrt{t}} \approx 13.0259\left(1-S^{4}\right)-1.62824\left(1-S^{4}\right)^{2}+0.3383\left(1-S^{4}\right)^{3}$.

4 order approximate solution

$\frac{x}{\sqrt{t}} \approx 13.0601\left(1-S^{4}\right)-1.63251\left(1-S^{4}\right)^{2}+0.34312\left(1-S^{4}\right)^{3}-0.08465\left(1-S^{4}\right)^{4}$.

5 order approximate solution

$$
\begin{aligned}
\frac{x}{\sqrt{t}} \approx & 13.05136\left(1-S^{4}\right)-1.63142\left(1-S^{4}\right)^{2}+0.34189\left(1-S^{4}\right)^{3}-0.084718\left(1-S^{4}\right)^{4} \\
& +0.022764\left(1-S^{4}\right)^{5}
\end{aligned}
$$

Compared finite element method (FEM) varying from 0 to $20 \mathrm{~mm}$ in the space coordinate $x$, which is divided into 480 units and calculated for results with saturation $S$ in $t=1 \mathrm{~min}$ as the exact solution, the results of $S$ obtained from Eq. 18 vs. FEM are shown in Table 1. The present method shows the results of 1-5 orders approximations of Eqs. 14-18 in Fig. 1.

Table 1: Results of $S$ obtained from FEM and present method ( $t=1 \mathrm{~min}$ )

\begin{tabular}{cccc}
\hline$x(\mathrm{~mm})$ & 5 order approximate solution & FEM & Relative error $(\%)$ \\
\hline 11.69881732 & 0.1 & 0.101 & $-0.9901 \%$ \\
11.68293136 & 0.2 & 0.195067 & $2.5289 \%$ \\
11.61404598 & 0.3 & 0.297302 & $0.9075 \%$ \\
11.42820963 & 0.4 & 0.398995 & $0.2519 \%$ \\
11.03453688 & 0.5 & 0.49962 & $0.0761 \%$ \\
10.31214123 & 0.6 & 0.59989 & $0.0183 \%$ \\
9.103210514 & 0.7 & 0.69998 & $0.0029 \%$ \\
7.198555699 & 0.8 & 0.800021 & $-0.0026 \%$ \\
4.308249764 & 0.9 & 0.90011 & $-0.0122 \%$ \\
0 & 1 & 1 & $0.0000 \%$ \\
\hline
\end{tabular}

In Table 1, the results of the 5 orders approximation solution are very close to the results of FEM. The maximum relative error value of present method is only $-0.9901 \%$ in $S=0.1$ for the 5 order approximation solution. 


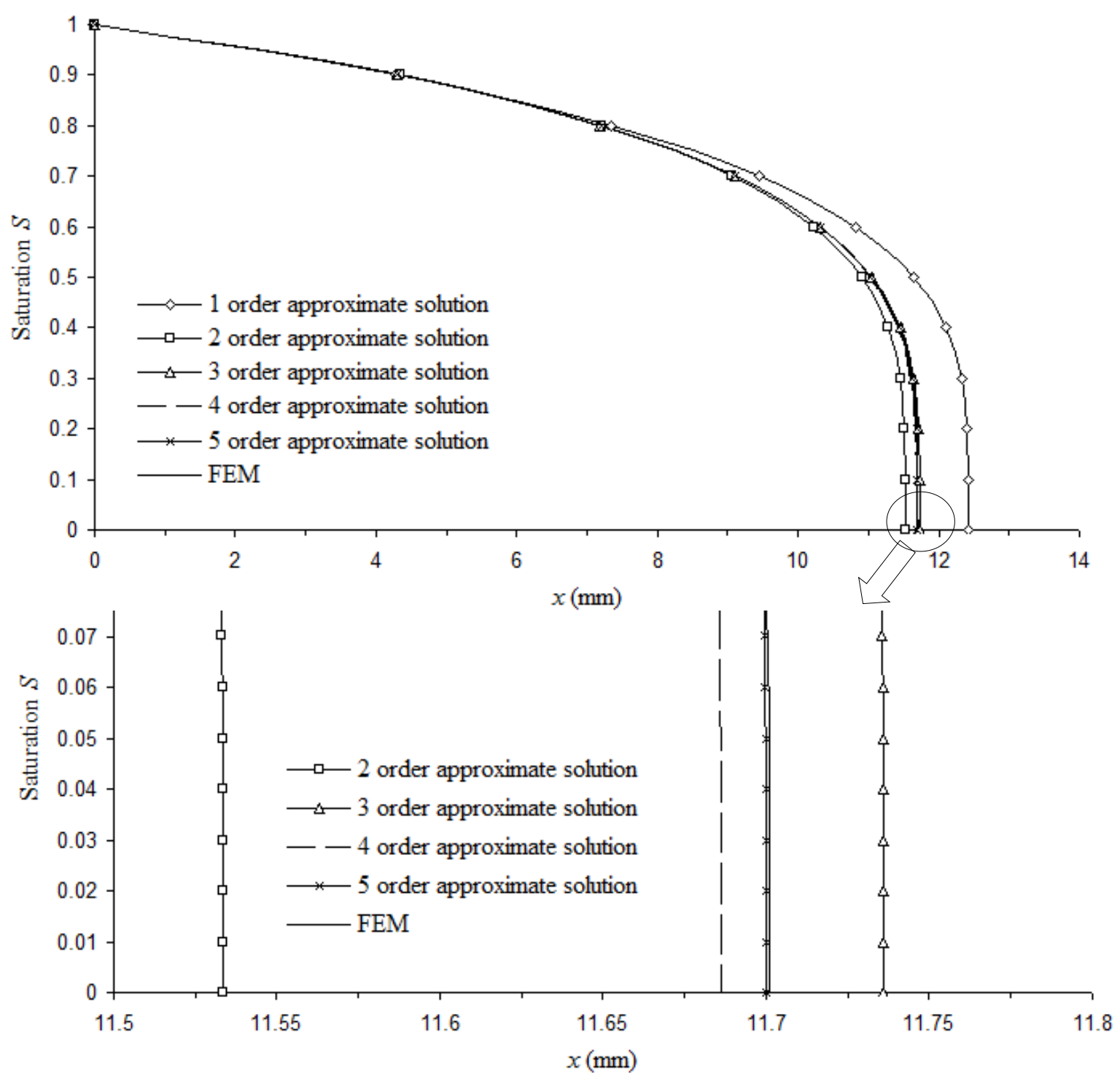

Fig. 1 Results of saturation $S$ obtained from FEM and present method in $t=1 \mathrm{~min}$

Noting Fig. 1, it can be seen that the saturation $S$ decreases from 1 to 0 as the space coordinate $x$ increases. Due to the effect of hydraulic diffusivity $D(S)$, the saturation $S$ decreases very fast from 0.4 to 0 when $x$ increases from 11 to $11.7 \mathrm{~mm}$. The solutions of the present method are closer to the exact solutions as the order $k$ increases from 1 to 5 in Fig. 1.

\section{Conclusion}

In this paper, one-dimensional approximation solution of RE is derived by applying Boltzmann transformation and term by term differentiation in Eq. 13. The hydraulic conductivity in RE owns Brooks-Corey model, and the initial condition parameter $S_{0}$ could be an arbitrary constant. The presented example demonstrates the accuracy of the present solution by comparing the present results with the results obtained from the FEM.

\section{Acknowledgments}

This work is supported by the National Natural Science Foundation of China (Grant no.11302119).

\section{References}

[1] M. B. Parlange, S.N. Prasad, J. Y. Parlange and M.J.M. Romkens: Water Resour. Res. Vol. 28 (1992), p. 2793

[2] N. Khodabakhshi, S.M. Vaezpour and D. Baleanu: Fract. Calc. Appl. Anal. Vol. 17 (2014), p. 382

[3] T. P. Witelski: Adv. Water Resour. Vol. 28 (2005), p. 1133 
[4] M. Omidvar, A. Barari, M. Momeni and D. D. Ganji: Geomech. Geoeng. Int. J. Vol. 5 (2010), p. 127

[5] D. Lockington, J.Y. Parlange and P. Dux: Mater. Struct. Vol. 32 (1999), p. 342

[6] M. T. van Genuchten: Soil Sci. Soc. Am. J. Vol. 44 (1980), p. 892

[7] R. Brooks, H.T. Corey: Hydraulic Properties of Porous Media (Colorado State University, Fort Collins, the United States of America 1964).

[8] C. Xi, D. Ying: Appl. Math. Mech. Vol. 37 (2016), p. 169 